\title{
The FIRST radio survey: Panchromatic properties of FIRST radio sources identified in Boötes and Cetus fields
}

\author{
K. El Bouchefry ${ }^{1, \star}$ \\ Astrophysics and Cosmology Research Unit, University of KwaZulu-Natal, Westville, 4000, South Africa
}

Received 2008 Apr 30, accepted 2008 Aug 19

Published online 2008 Dec 28

Key words galaxies: high-redshift- radio continuum: galaxies - infrared: galaxies-surveys - galaxies: evolution - galaxies: starburst - galaxies:formation

In this paper, the second in a series, the availability of multi-wavelength optical/infrared information of FIRST (Faint Images of the Radio Sky at $20 \mathrm{~cm}$ ) radio sources counterparts over $\sim 9.2 \mathrm{deg}^{2}$ in Boötes field and $\sim 2.4 \mathrm{deg}^{2}$ in Cetus field is exploited to infer the physical properties of the faint radio population. The radio sources optically identified have been divided into resolved galaxies and stellar-like objects finding that the faint radio population is mainly composed of early-type galaxies with very red colour $(B w-R \sim 4.6)$. A total number of 57 counterparts of FIRST radio sources have extremely red colour $(R-K \geq 5)$. Photometric redshift from Hyperz implies that the Extremely Red Objects (EROs) counterparts to FIRST radio sources are mostly located in the range $z=0.7-2$, with the bulk of the population at $z \sim 1$. Taking advantage of the near infrared imaging with FLAMEX (FLAMINGOS Extragalactic Infrared Survey), the EROs counterparts to FIRST radio sources are separated into passively-evolving and dusty star-forming galaxies using their $R J K$ colours; the relatively blue $J-K$ of these galaxies suggest that most are old elliptical galaxies (18/25) rather than dusty starburst (7/25). A total of 15 Distant Red Galaxy (DRGs) have been identified as counterparts to FIRST radio sources in Cetus field and 3 DRGs in Boötes field with $J-K>2.3$.

(C) 2006 WILEY-VCH Verlag GmbH \& Co. KGaA, Weinheim

\section{Introduction}

The radio sources in deep radio surveys at $1.4 \mathrm{GHz}(20 \mathrm{~cm})$ consist of two main populations; active galactic nuclei and star forming galaxies (Condon 1984, Windhorst et al. 1985). It has long been known that powerful radio sources are associated with AGN or Giant elliptical (e.g. Windhorst 1990). At mJy level and fainter, deep radio surveys at $1.4 \mathrm{GHz}$ (Condon 1984, Windhorst et al. 1985. Condon 1992) at mJy level and fainter have shown that the faint radio population is a mixture of different classes of objects (radio loud, and radio quite AGN, starburst galaxies, spirals). To date the nature and the level of contribution of each population is still not well established, and very little is known about the cosmological evolution of different kinds of objects and so many other topics despite so many studies (e.g. Georgakakis et al. 1999; Gruppioni et al. 1999; Ciliegi et al. 2003; Hopkins et al. 2003; Prandoni et al. 2006; Fomalont et al. 2006; Simpson et al. 2006; Bondi et al. 2007). This is mainly due to the incompleteness of optical identification and the optical depth of the spectroscopic follow up. Therefore, large and statistically complete and homogeneously selected samples, very deep optical imaging and spectroscopic follow up are required for reasonably large deep radio samples in order to investigate the nature and evolution of the faint radio population.

\footnotetext{
^ e-mail: kelbouchefry@gmail.com
}

Studies of Extragalactic radio sources are among the most interesting challenges of modern cosmology and astrophysics. These studies have been invigorated due to the recent generation of panchromatic photometric and spectroscopic large area surveys, such as NVSS (Condon et al. 1998), FIRST (Becker et al. 1995), SDSS (York et al 2000), and 2DF (Colless et al. 2001). These surveys have provided additional panchromatic photometric and optical/infrared spectroscopic observations (Sadler et al. 1999, Best et al. 2005). For example, Ivezic et al. (2002), Best et al. (2005) and Obric et al. (2006) cross correlated FIRST and SDSS, and analysed the optical and radio properties of quasars and galaxies in full details. Best (2004) studied the environmental dependence of radio luminous AGN and Star Forming galaxies, and their luminosity function has been investigated in other studies (Sadler et al. 1999, Jackson \& Lindish 2000, Chan et al. 2004, Best et al. 2005). Recent searches have been undertaken to search for clustering of galaxies (Hall et al. 2001) and extremely red objects (EROs) around high redshift quasars and radio galaxies (Cimatti et al. 2000, Wold et al. 2003, Zheng et al. 2005, and references therein). EROs are of special interest in the study of galaxy evolution, in that their colours and other properties suggest that they are the high-redshift $(z=1-2)$ counterparts and progenitor of local elliptical and So galaxies, and are amongst the oldest galaxies present at these redshifts.

The present paper is the second in a series analysing the properties of the FIRST radio sources which are iden- 
tified in NDWFS/FLAMEX surveys. In paper I, the author has identified FIRST radio sources in Boötes field over a region of $\sim 9.2 \mathrm{deg}^{2}$ and in Cetus field over a region of $\sim$ $4.2 \mathrm{deg}^{2}$. The identification rate was found to be $63 \%$ in $\mathrm{Bw}$ band, $65 \%$ in $\mathrm{R}$ band, $64 \%$ in I band, $38 \%$ in $\mathrm{K}$ band and $39 \%$ in four bands ( $B w, R, I$ and $K$ ). I also derived photometric redshifts of FIRST radio sources counterparts using the public code Hyperz. In this paper (II) I want to shed some light into the optical/infrared properties of the FIRSTBoötes/Cetus sample and their environment.

The layout of this paper is as follows: Section 2, presents the magnitude-flux distribution of the FIRST counterparts in Boötes and Cetus field. Section 3 discusses the properties of FIRST radio sources in Cetus field. Section 4 investigates the EROs counterparts to FIRST radio sources and section 5 summarises conclusions.

Throughout this paper it is assumed that $\mathrm{H}_{\circ}=70 \mathrm{~km} \cdot \mathrm{s}^{-1}$ $\mathrm{Mpc}^{-1}, \Omega_{M}=0.3$, and $\Omega_{\Lambda}=0.7$ unless stated otherwise.

\section{Magnitude - Flux distribution in Boötes field}

The combination of the sample introduced in El Bouchefry \& Cress 2007 (hereafter EC07) and those introduced in paper I (El Bouchefry 2008) provided a total of 338 FIRST radio sources with infrared counterparts and a total of 273 sources with identification in four bands $(B w, R, I, K)$. The FIRST radio sources with optical identifications have been divided into galaxies and stellar objects by means of the SExtractor Class-Star parameter S/G (Bertin \& Arnouts 1995). This parameter is provided by the NDWFS catalogue and ranges from 0.0 to 1.0 being the most point like. Of the 273 IDs, 243 sources were identified as galaxies and 30 sources identified as stellar objects. Combining the analysis of the radio and photometric properties can provide a first indication of the nature of the faint radio population. Figure 1 shows the $20 \mathrm{~cm}$ radio flux versus B, R, I and $\mathrm{K}$ magnitudes for all FIRST radio sources with an optical/infrared identifications. Superimposed are the lines corresponding to constant values of the observed radio-to-optical ratio $r$, defined by Condon (1980) as follows:

$$
r=S \times 10^{0.4(\operatorname{mag}-12.5)}
$$

where $S$ is the $1.4 \mathrm{GHz}$ flux in mJy and mag is the apparent magnitude of the optical/infrared counterparts.

In all diagrams empty squares stand for resolved sources (galaxies), filled diamonds for unresolved sources (stellar objects) and filled circles represent a radio-loud quasar (J142738.5+3312) discovered by McGreer et al. (2006) as a counterpart to FIRST radio sources by combining NDWFS and FLAMEX data. A radio-loud source is considered to have $r>10$ (Urry \& Padovani 1995). Magliocchetti, Celotti \& Danese (2002) showed that for star-burst galaxies, $r_{B}<100$. Therefore, the $B w, R, I, K$ magnitude flux diagrams (Figure 1) show that there are a few star-forming
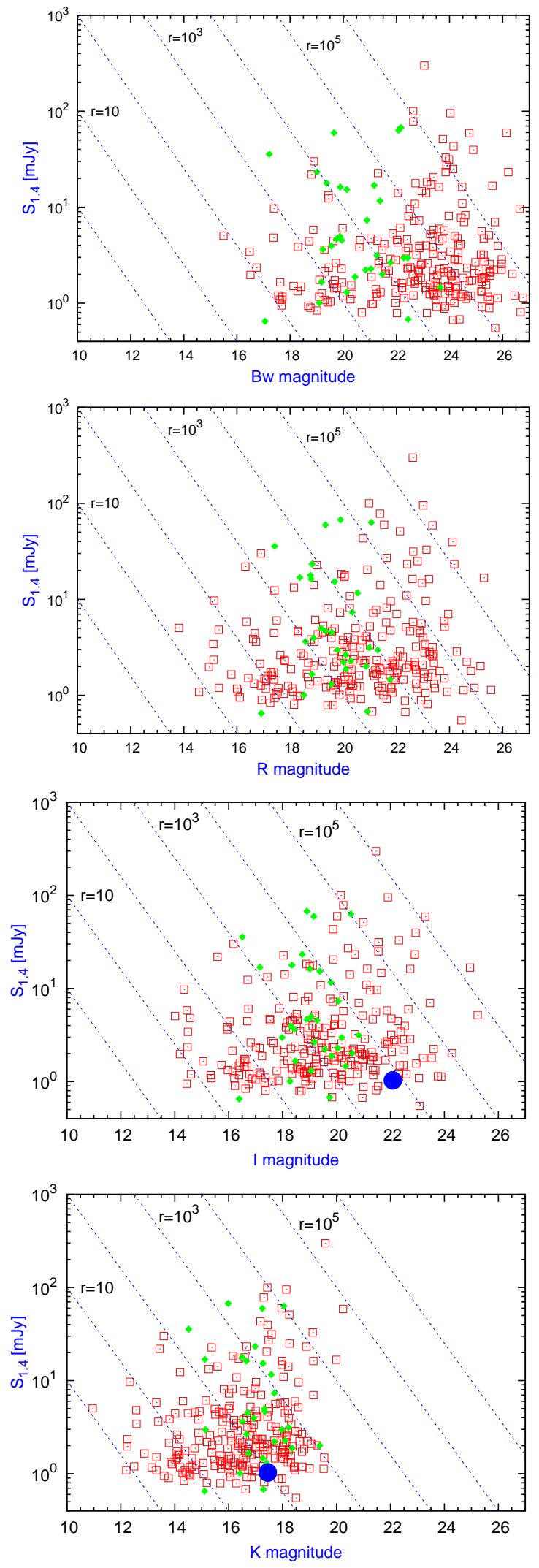

Fig. 1 The radio flux vs. $B w, R, I$ and $K$ magnitudes for all FIRST radio sources identified in Boötes field. Empty squares represent sources identified as galaxies, filled diamonds indicates sources identified as stellar-like objects and the large filled circle (in I and $\mathrm{K}$ bands) represents a radio-loud quasar (McGreer et al. 2006). Superimposed are the lines corresponding to constant values of the radio-tooptical ratio $r=10,100,10^{3}, 10^{4} \ldots 10^{6} \quad$ www.an-journal.org 


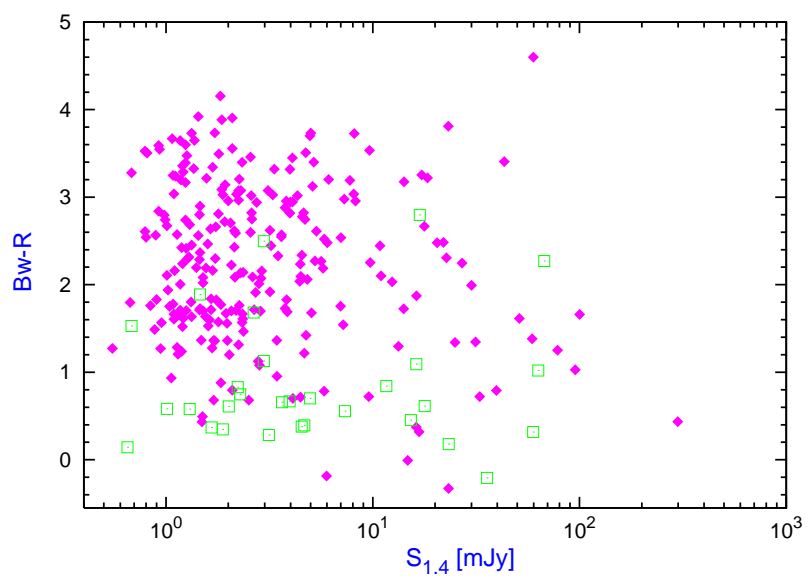

Fig. 2 The Colour Bw-R versus radio flux for all FIRST radio sources counterparts. Filled diamonds indicate sources identified as galaxies while empty squares represent stellar-like objects

galaxies in the FIRST-NDWFS/FLAMEX sample down to $1 \mathrm{mJy}$. This result may indicate that star-forming galaxies actually dominate the sub-mJy population. This is also reported by Gruppioni et al. 1999b, in the study of the Marano field, Georgakakis et al. (2005) in the Phoenix survey and Magliocchetti et al. (2002) in the FIRST-APM sample.

It is important to note how the radio population of radio galaxies is shifting towards brighter red magnitudes (see Figure 1). The shift is observed especially for resolved sources (galaxies) and not for the stellar identifications, highlighting the fact that faint radio galaxies are typically red, early-type objects. Figure 2 represents the $B w-R$ colour as a function of radio flux $\mathrm{S}_{1.4}$ for the two populations: resolved sources (filled diamonds) and stellar objects (empty squares). It clearly seen see from the plot that, the majority of FIRST radio sources counterparts consists of galaxies with very red colours, up to $B w-R \sim 4.6$. One also notes that, the radio galaxies appear mostly with $B w-R>1$, and stellar-like objects dominate the region $0<B w-R<1$ leaving a very small fraction of objects with blue colour $(B w$ $-R<0$ ) characteristic of star forming galaxies. Again this is a further confirmation of the results of Magliocchetti \& Maddox (2002) and Magliocchetti et al. (2004) in the radiooptical study of the FIRST radio sources in APM and 2DF surveys respectively.

\section{Properties of FIRST radio sources in Cetus field}

Cross correlating the FIRST radio sources with sources in Cetus field yielded 113/242 counterparts in $J$ band; 124 radio sources identified in $K$ band and 109 sources identified in two bands ( $J, K$, see El Bouchefry 2008).

Figures 3 and 4 illustrate the $J$ - $K$ colour against $K$ magnitude and photometric redshift respectively. A total of 15

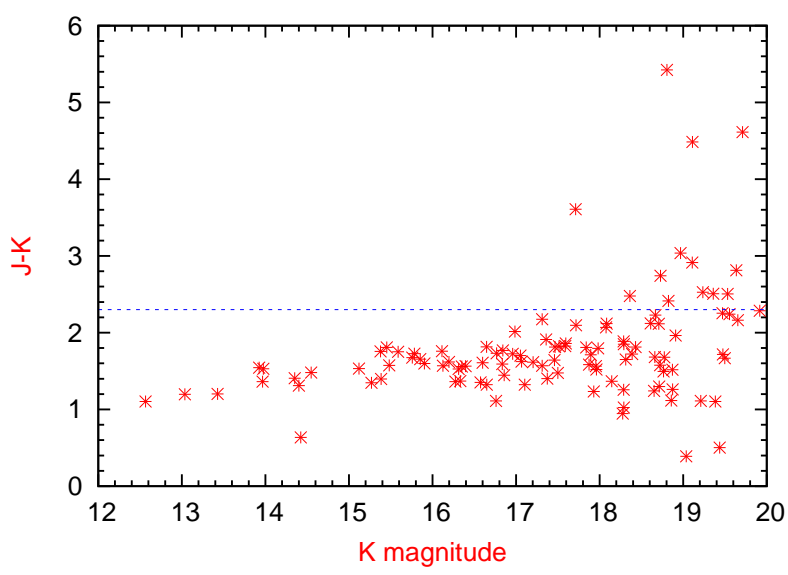

Fig. 3 The Colour magnitude sequence for all FIRST radio sources identified in Cetus field.

counterparts to FIRST radio sources in this field have a red colour $J-K>2.3$ that selects the so-called Distant Red Galaxies (DRGs). This latter in turn select an heterogeneous samples of galaxies going from the passively evolving systems to a significant fraction of obscured star forming galaxies at $2<z<4.5$ with strong Balmer or $4000 A^{\circ}$ breaks. As a comparison, Hall et al. (2001) select four galaxies with $J-K>2.5$ and found photometric redshift $z \geq 2$, Franx et al. (2003) found 14 DRGs with $J-K>2.3$ in a photometric redshift range from 1.92 to 4.26 (see also Takagai et al. 2007, Papovich et al. 2006).

In the FIRST - Cetus sample, there are 3/15 DRGs counterparts to FIRST radio sources at lower redshift $(1 \leq z \leq$ 2 ) , and $8 / 15$ with photometric redshift ranging from 2 to 3.8 and $17<K<20$. Papovich et al. (2006) reported that lower redshift objects are dominated by dusty star bursts and higher redshift are objects with more complex stellar population, these are likely to be passively evolving stellar components. Obviously spectroscopic confirmation is required before one can draw any conclusion. Figure 5 illustrates the magnitude - flux diagram for the FIRST radio sources identified in $\mathrm{J}$ (empty squares) and $\mathrm{K}$ (filled diamonds) bands. It is noted again an interesting shift of the population towards brighter magnitude with a small number of sources that have a radio-to-optical ratio in $J$ band $r \leq 100$.

\section{Extremely red objects counterparts to FIRST radio sources in Boötes field}

Extremely red objects were first discovered more than 20 years ago (Elston et al. 1988) as resolved galaxies with $K \sim$ 16.5 and $R-K \sim 5$. Photometry and spectroscopy showed that these objects were old elliptical galaxies at $z=0.8$ (Elston et al. 1989). EROs seem to be found everywhere; they were found in the vicinity of high redshift ( McCarthy et al. (1992); in a quasar field (Stockton et al. 2006; Hu \& Ridgway 1994), as counterparts of faint X-ray (Newsam et al. 1997) and as a counterparts of radio sources (Smail et al. 


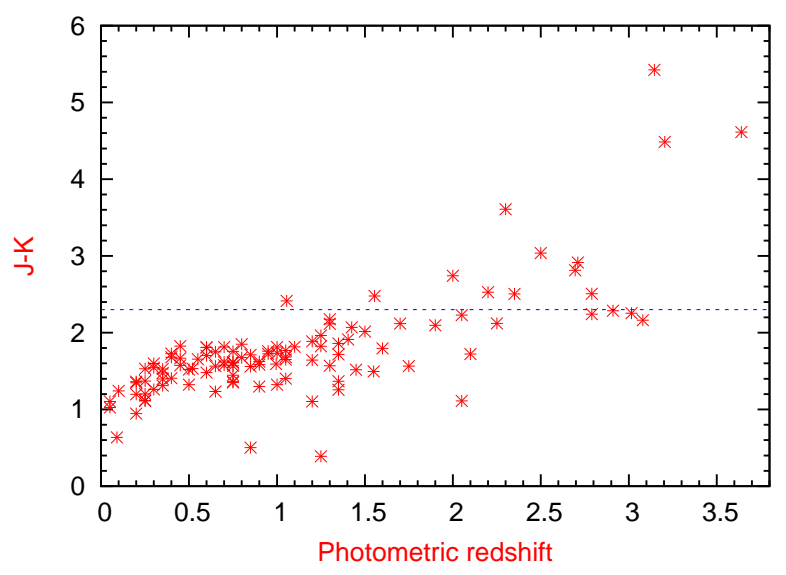

Fig. 4 The Colour $J-K$ against photometric redshift for all FIRST radio sources identified in Cetus field.

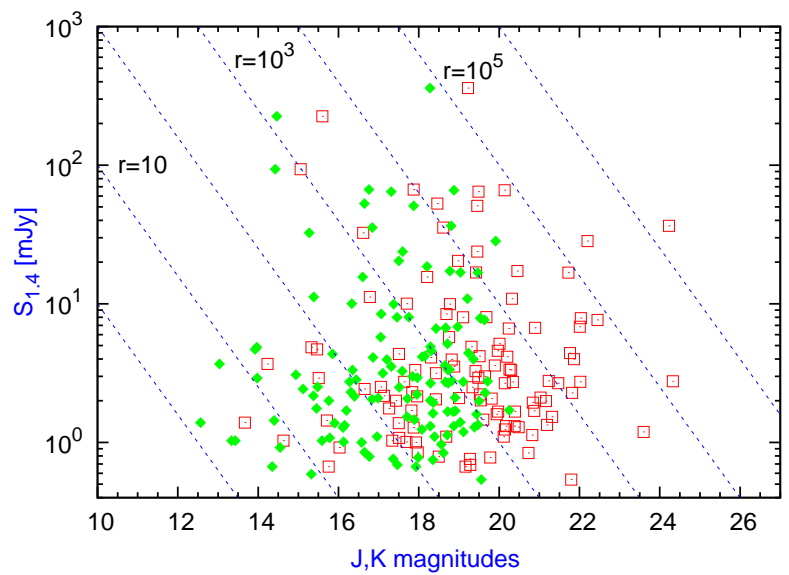

Fig. 5 The radio flux vs. the magnitude for FIRST radio sources identified in Cetus field, in $J$ band (empty squares) and $K$ band (filled diamonds). Dashed lines correspond to constant values of the radio-to-optical ratio $R=$ $10,100,10^{3}, 10^{4} \ldots 10^{6}$

2002; Willott, Rawlings \& Blundell 2001, De Breuck et al. 2001, Spinrad et al. 1997). Their colours are consistent with two heterogenous mix of galaxy classes: 1) Old, passively evolving elliptical galaxies at $z \geq 0.9$, the colour is due to the lack of star formation and the large K-Correction, 2) dust reddened star-forming galaxies or AGN (see e.g. Cimatti et al. 2003; Wold et al. 2003; Yan \& Thompson 2003; Daddi et al. 2002; Smail et al. 2002; Roche et al. 2002). The identification of either type offers a potentially important deep insight into the formation and evolution of elliptical galaxies and help to investigate the existence of a population of dusty galaxies of AGN strongly reddened by dust extinction.

Several selection criteria have been defined for EROs, including $R-K \geq 6, R-K \geq 5.3, R-K \geq 5, I-K \geq 4$ with K-magnitude upper limits from 18 to $21 \mathrm{mag}$. All these criteria are designed to find evolved galaxies at high redshift (Cimatti et al 1999, Abraham et al. 2004, Pozzetti \& Manucci 2000). The limit of $R-K=5$ is adopted in this study as the definition of an ERO. Based on this 57 (19\%) FIRST radio sources are found to have an ERO as a counterparts with $R-K \geq 5$ and $16.5 \leq K \leq 20$. This fraction includes sources that have identifications in five bands (Bw, R, I, J, K), four bands (Bw, R, I, K) or less (i.e. three bands $((\mathrm{Bw}, \mathrm{R}, \mathrm{K}) ;(\mathrm{R}, \mathrm{I}, \mathrm{K})$ or two bands $(\mathrm{R}$, $\mathrm{K})$ ). As a comparison with ERO samples selected based on the criteria $(I-K) \geq 4$, I found 41 EROs and 33 of the $(R-K) \geq 5$ EROs have $(I-K) \geq 4$. The $\mathrm{R}$ and $\mathrm{I}$ bands based selection criteria for EROs are not equivalent. Figure 6 shows the $R-K$ vs. $K$ (left panel) and $I-K$ vs $K$ (right panel) colour-magnitude diagram respectively for FIRST radio sources with IDs in both R, K bands (305). This figure shows that there are no stars (empty diamonds) that have a colour redder than $R-K \geq 5$. 30/57 sources appear to have a magnitude $\leq 18$ and $27 / 57$ with a magnitude $>18$. Figure 7 shows the $K$ band magnitude distribution (left panel) and photometric redshift distribution (right panel) of all 57 EROs. The median $K$ band magnitude of these 57 EROs is $\mathrm{K}=19.27 \pm 0.72$ and is equal to $\mathrm{K}=17.97 \pm 0.71$ when sources with $z>2$ excluded.

\subsection{Colour-colour separation}

Since EROs are composed of both passively evolving red galaxies and dusty starburst, colours are one means of breaking the degeneracy between these two groups. The SED of an elliptical galaxy at $z \sim 1$ drops off sharply at the $4000 A^{\circ}$ between $R$ and $K$ bands, while the SED of a dusty starburst declines more gradually because of reddening. Therefore observations between the $R$ and $K$ bands, such as $I, Z, J$ or $H$ can be used to measure the sharpness of the spectral type break and thus discriminate between these two scenarios (Pozzetti \& Mannucci (2000)). The $(J-K)$ separates EROs at $z>1$ to bluer early type galaxies and to redder dusty EROs. As there is no J band for all FIRST radio sources IDs; I used only the EROs counterparts of FIRST radio sources identified in the second strip $\left(33^{\circ} \leq \delta<34^{\circ}\right)$ where the $\mathrm{J}$ band data are available from FLAMEX. There are 30 EROs in this strip where 25/30 were detected in $\mathrm{J}$ band.

Figure 8 shows $R-K$ versus $J-K$ colour-colour scheme introduced by Pozzetti \& Mannucci (2000), where elliptical galaxies lie in the left (bluer $J-K$ ), and dusty starburst lie to the right (redder $J-K$ colour). I find $7 / 25$ (18\%) EROs selected in the dusty starburst side of the indicator and 18/25 (72\%) appear elliptical. The colour-colour scheme introduced by Bergstrom \& Wiklind (2004) using the $(R-J)$ colour vs. $(J-K)$ colour is also used. There is a total agreement with Pozzetti \& Mannucci (2000) scheme. But no reasonable agreement was found between the classification using the SED template fitting method and the optical/near-infrared colours of the sample. There are 11 sources (with $\chi^{2}<2.7,90 \%$ confidence limit ) classified as burst using Hyperz and classified as elliptical in the colourcolour diagram (see following section). 

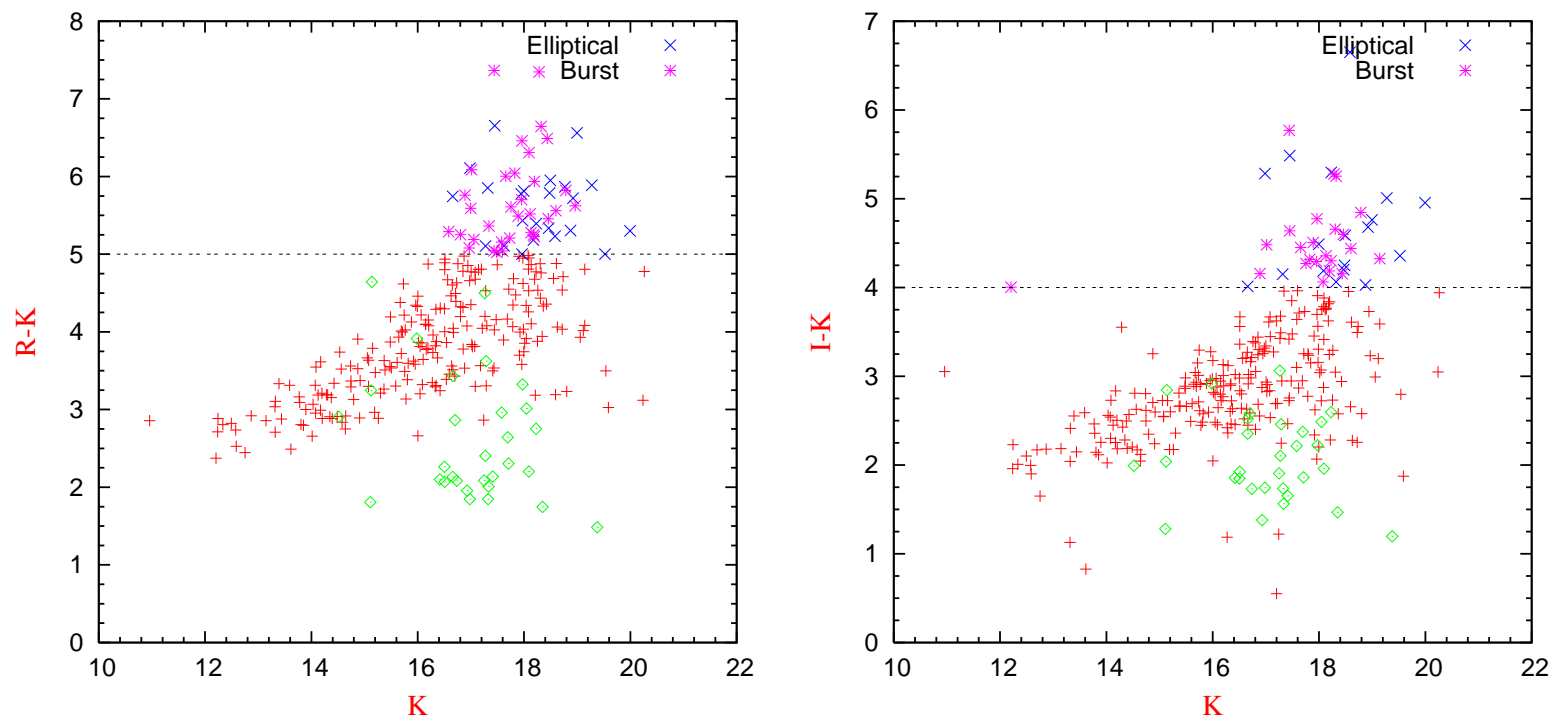

Fig. 6 The $\mathrm{K}$ band magnitude vs. the $R-K$ and $I-K$ colours, in left and right panels, respectively. Empty diamonds indicate stellar-like sources, while the crosses, stars and plus signs represent objects identified as galaxies. The horizontal long-dashed line corresponds to the limit adopted for the selection of the sample of EROs counterparts to FIRST radio sources in Boötes field.
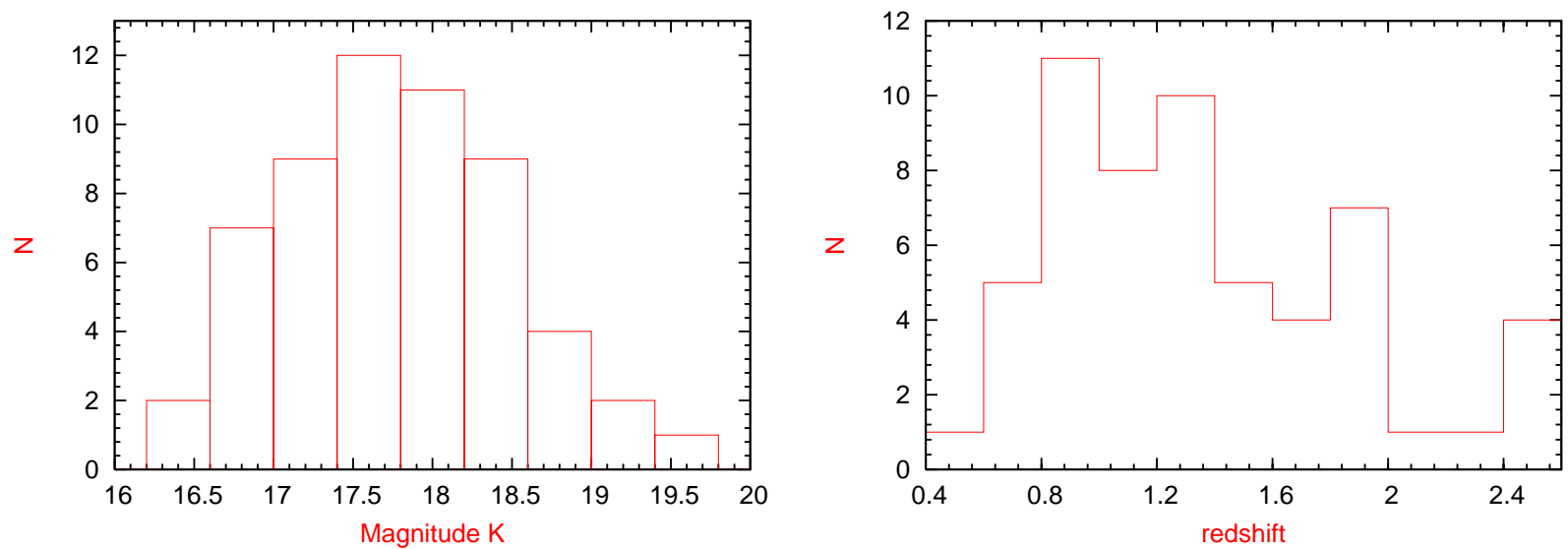

Fig. 7 The photometric redshift and K band magnitude distribution for the 57 EROs counterparts (in Boötes field) in left and right panels respectively.

\subsection{Photometric classification of EROs}

As a second way to classify an EROs sample to whether passively evolving red galaxies, or dusty starburst galaxies, I use the same method described by Smail et al. (2002). The Hyperz code was used to find the best-fit ( $\chi^{2}$ minimum); empirical galaxy template by comparing the EROs colours to two different families of SEDs and sought the best solution: dusty, young starburst (or AGN) or almost dust free evolved systems. All models in Hyperz are built with GISSEL 98 (which is an update of the spectral synthesis models described by Bruzual \& Charlot (1993)) with Miller \& Scalo (1979) initial mass function, solar metalicity and different star formation rate (SFR). For the dusty starburst I adopted a continuous star formation model and the redden- ing $\left(A_{v}\right)$ ranges between 1 and 4 (Cimatti et al 2002). While for the evolved galaxies I used a model SED represented by exponentially decaying star formation rate with e-folding time of $1 \mathrm{Gyr}$, the reddening for this model is required to be $A_{v}<0.5$. For these model fits I adopted the Calzetti et al. (2000) reddening law. Since I do not have J band data for all the sources, the Hyperz was run for two samples: the first sample included only EROs counterparts of FIRST that have $\mathrm{J}$ band data (provided by FLAMEX; $33^{\circ} \leq \delta<34^{\circ}$ ), there are 25/57 sources with $\mathrm{J}$ data and 20/25 detected in 5 bands $(B w, R, I, J, K)$. The second sample contains $27 / 57$ that do not have $\mathrm{J}$ band data (third and fourth strips; $34^{\circ} \leq \delta<36^{\circ}$ ) and $17 / 27$ identified in four bands ( $\mathrm{Bw}, \mathrm{R}$, I, K). The photometric redshift predictions of three fits (Ell, Burst, all BC SED templates (E, Burst, SO, Sa, Sb..)) are 


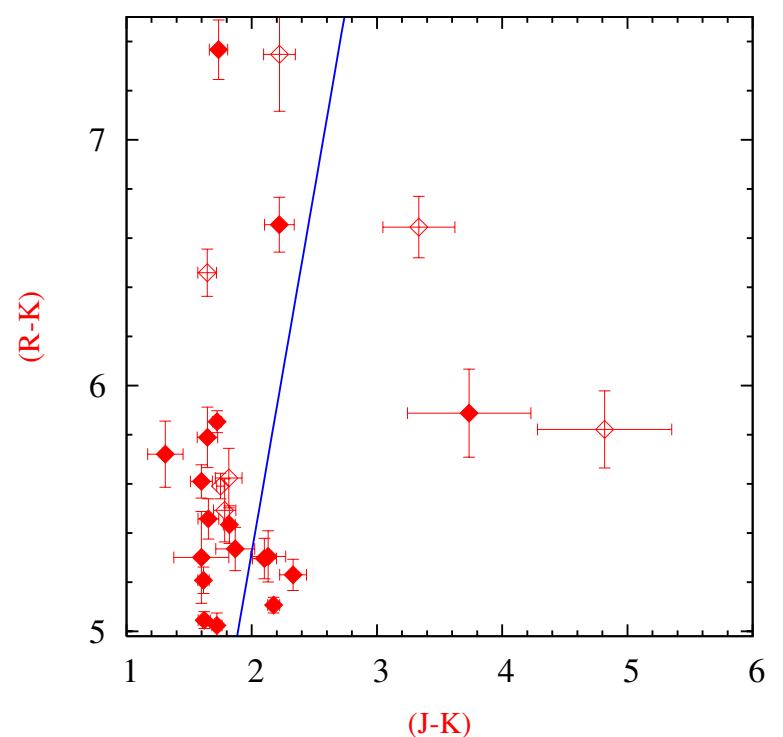

Fig. $8 R$ - $K$ against $J-K$ Colour-colour diagram for the 25 counterparts of FIRST radio sources that have $J$ band (provided by FLAMEX) with $R-K>5$. The solid line represents the boundary between dusty starburst and evolved, passive magnitude EROs proposed by Pozzetti \& Mannucci (2000); the dusty galaxies should lie to the right of the line (redder $(J-K)$ colours) with the evolved systems on the left.

shown in table 1 One notes that, the best-fit elliptical template has a lower photometric redshift than the best-fit starburst template. The mean photometric redshift is $z=1.35$ and the rms scatter in the photometric redshift predictions for these three fits is $\sigma_{z}<0.51$; the photometric redshifts are thus quite robust and show clearly that these EROs are $z \geq 0.8$ galaxies (except two sources one at $z=0.605$ classified as a dusty source using the colour-colour plot and the second one at $z=0.55$ classified also as dusty source according to the template fitting with a high probability). When all the SED templates are allowed; 14/57 sources cannot provide an acceptable fit giving $\chi^{2}>2.7 ; 11 / 57$ are classified as elliptical and $31 / 57$ as starburst. When the elliptical option is chosen $28 / 57$ has $\chi^{2}<2.7$ (90\% confidence limit) and $22 / 57$ has $\chi^{2}>2.7$ when the starburst option is chosen. It is important to note that broad band photometry alone cannot provide a reliable information on the spectral type due to the degeneracy between age, metalicity and reddening (see Fig 1 in Bolzonella, Miralles \& Pello 2000). Nevertheless it provides a rough SED classification at two extreme: a given object has "blue " or "red" continuum at a given $z$. The early type galaxies can be matched with elliptical or burst templates. These SEDs are nearly identical (Bolzonella, Miralles \& Pello, 2000) and the best-fit Hyperz 'starburst' are evolved results of instantaneous burst and not really dusty star-forming galaxies. Table 1 presents the object name; $1.4 \mathrm{GHz}$ radio flux density, colours $(R-K$ and $J-K)$, the $K$ band magnitude, Photometric redshift and best fitting SED $\left(\chi^{2}\right)$ (either, "starburst", "elliptical") of all EROs counterparts of FIRST radio sources. One of the dusty sources is assigned redshift $z<0.8(z=0.605)$ lower than that expected for EROs.

\section{5 conclusions}

In this paper I have discussed the nature of the faint radio population at the mJy level by making use of a sample of $\sim 700$ objects drawn from the joint use of the FIRST survey and the NDWFS/FLAMEX surveys.

I find that the population of faint radio sources is mainly dominated by early type galaxies, with radio to optical ratio between $10^{2}$ and $10^{6}$ and a very red colour $B w-R \sim 4.6$ confirming the results found by Magliocchetti et al. (2002) who studied the optical counterparts of FIRST radio sources in the APM survey to a limiting magnitude of $\mathrm{B} \sim 21$ and $R \sim 20$.

I find 13 DRGs counterparts to FIRST radio sources in Cetus field with $J-K>2.3$ to $K<20$. Obviously deep optical imaging and spectroscopy is required to investigate the nature of these DRGs.

Taking advantage of the infrared data provided by FLAMEX survey $\mathrm{J}$ and $\mathrm{K}$ bands, I find 57 EROs counterparts to FIRST radio sources (in Boötes field) with $R-K \geq$ 5 . I make use of a $J-K$ vs. optical infrared colour-colour diagram (Pozzetti \& Mnuucci 2000) to separate EROs into passively evolving and dusty star-forming galaxies finding that there is $18 / 25(72 \%)$ of EROs are early type galaxies with $z=0.6-2$ and $7 / 25(28 \%)$ dusty star forming galaxies. $3 / 7$ EROs from this latter group are DRGs with $J-K>2.3$.

Using the NDWFS/FLAMEX deep $B w R I J K$ imaging I photometrically classify the EROs counterparts to FIRST radio sources into dusty or evolved based on simple SED models. The latter one cannot really provide a reliable information about the spectral type due to the degeneracy between age, metalicity, but provides rough SED classification at two extremes either "blue" or "red" (Bolzonella, Miralles \& Pello, 2000).

Finally, Spectroscopic follow up of the faint radio population and their surrounding EROs (and DRGs), deeper optical and infrared imaging is required in order to establish colours for the faint radio population and explore their morphology in more detail. Deeper optical/infrared imaging will also allow to select deeper and more complete sample of EROs and to determine the relationship between the radio galaxies and the EROs allowing for a more detailed study of the faint radio population environment. It is proposed to follow up the southern field (Cetus) spectroscopically using the Southern African Large Telescope.

Acknowledgements. I would like to thank the anonymous referee for helpful comments and suggestion which improved the paper. I would like to thank Dr Gonzalez Antony for answering all the questions concerning the FLAMEX surveys. 
I also would like to thank South African Square Kilometre Array project for supporting and funding my Ph.D studies.

This work makes use of images data products provided by the NOAO Deep Wide-Field Survey (Jannuzi and Dey 1999), which is supported by the National Optical Astronomy Observatory (NOAO). NOAO is operated by AURA, Inc., under a cooperative agreement with the National Science Foundation.

This publication makes use of data products from the FLAMEX survey. FLAMEX was designed and constructed by the infrared instrumentation group (PI: R. Elston) at the University of Florida, Department of Astronomy, with support from NSF grant AST97-31180 and Kitt Peak National Observatory.

\section{References}

Abraham, R.G., Glazebrook, K., McCarthy, P.J.: 2004, AJ 127, 2455

Becker, R. H., White, R. L., Helfand, D. J.: 1995, ApJ 450, 559

Bergstrom, S., Wiklind, T.: 2004, A\& A 414,95

Bertin, E., Arnouts, S. : 1996, A\& AS 117, 393

Best, P.N. : 2004, MNRAS 351, 70

Best, P.N., Kauffmann, G., Heckman, T.M., Ivezic, Z.: 2005, MNRAS 362, 9

Bolzonella, M., Miralles, J.-M., Pello, R. : 2000, A\& A 363, 476

Bondi, M., Ciliegi, P., Venturi, T. et al.: 2007, A\& A 463, 519

Bruzual, A. G., Charlot, S.: 1993, ApJ 405, 538

Calzetti,D., Armus, L., Bohlin, R.C., Kinney, A.L., Koorneef, J., Storchi-Bergmann, T. : 2000, ApJ 533, 682

Chan, B.H.P., Cram, L.E., Sadler, E.M., Killeen, N.E.B., Jackson, C.A., Mobasher, B., Ekers, R.D. : 2004, MNRAS 352, 1245

Ciliegi, P., Zamorani, G., Hasinger, G., Lehmann, I., Szokoly, G., Wilson, G.: 2003, A\& A 398, 901

Cimatti, A., Villani, D., Pozzetti, L., di Serego Alighieri, S.: 2000, MNRAS 318, 453

Cimatti, A., Daddi, E., di Serego Alighieri, S. et al.: 1999, A\&A 352,45

Cimatti, A., Daddi, E., Cassata, P. et al. : 2003, A\& A 412, L1

Colless, M., Dalton, G., Maddox, S. et al. : 2001, MNRAS 328, 1039

Condon, J.J.: 1984 , ApJ 287, 461

Condon, J.J.: 1992 , ARA\& A 30, 575

Condon, J. J., Cotton, W. D., Greisen, E. W., Yin, Q. F., Perley, R. A., Taylor, G. B., Broderick, J. J.: 1998, AJ 115, 1693

Daddi, E., Cimatti, A., Broadhurst, T. et al.: 2002, A\& A 384, L1

De Breuck, C.D., Breugel, W.V., Stanford, S.A. : 2001, AJ 123, 637

El Bouchefry, K., Cress, C.M. : 2007, AN 326, 577

El Bouchefry, K.: 2008, submitted

Elston, R., Rieke, G. H., Rieke, M. J.: 1988, ApJ 331, L77

Elston, R., Rieke, M. J., \& Rieke, G. H.: 1989, ApJ 341, 80

Fomalont, E. B., Kellermann, K. I., Cowie, L. L., Capak, P., Barger, A. J., Partridge, R. B., Windhorst, R. A., \& Richards, E. A.: 2006, ApJS 167, 103

Franx, M., Labbé, I., Rundnick G., et al.: 2003, ApJ 587, L79

Georgakakis, A., Mobasher, B., Cram, L., Hopkins, A., Lidman, C., Rowan-Robinson, M.: 1999, MNRAS 306, 708

Georgakakis, A., Afonso, J., Hopkins, A. M., Sullivan, M., Mobasher, B., \& Cram, L. E.: 2005, ApJ 620, 584

Georgakakis, A., Hopkins, A.M., Afonso, J., Sullivan, M., Mobasher, B. Cram, L.E. : 2006, MNRAS 367, 331.

Gruppioni, C., Mignoli, M., Zamorani, G.: 1999a, MNRAS 304, 199
Gruppioni, C., Mignoli, M., Zamorani, G.: 1999b, MNRAS 176, 365

Hall, P.B, Sawicki, M., Martini, P. et al.: 2001, AJ 121, 1840

Hopkins, A.M., Mobasher, B., Cram, L.E., Rowan-Robinson, M.: 1998, MNRAS 296, 839

Hu, E. M., Ridgway, S. E.: 1994, AJ 107, 1303

Ivezic, Z., Menou, K., Knapp, G.R. et al. : 2002, AJ 124, 2364

Jackson, C., Londish, D.M.: 2000, Publications of the Astronomical Society of Australia 17, 234

Magliocchetti, M., Maddox, S. J., Hawkins, Ed. et al.: 2004, MNRAS 350, 1485

Magliocchetti, M., Maddox, S.J.: 2002, MNRAS 330, 241

Magliocchetti, M., Celotti, A. , Danese, A.: 2002, MNRAS 329, 377

McGreer, Ian.D, Becker, R.H, David, J.H, White, R.L :2006, ApJ 652, 157

McCarthy, P.J., Persson, S.E., West, S.C.: 1992, ApJ 386, 52

Miller, G.E, Scalo, J.M.: 1979, ApJS 41, 513

Newsam, A.M., McHardy, I.M., Jones, L.R., Mason, K.O.: 1997, MNRAS 292, 378

Obric, M., Ivezic, Z., Best, P.N. et al.: 2006, MNRAS 370, 1677

Papovich, C., Moustakas, L.A., Dickinson, M., et al.: 2006, AJ 640, 92

Pozzetti, L., Mannucci, F.: 2000, MNRAS 317, L17

Prandoni, I., Gregorini, L., Parma, P., de Ruiter, H. R., Vettolani, G., Zanichelli, A., Wieringa, M. H., Ekers, R. D.: 2001, A\&A 369,787

Prandoni, I., Parma, P., Wieringa, M.H, de Ruiter, H.R, Gregorini, L., Mignano, A., Vettolani, G. Ekers, R.D. : 2006, A\& A 457, 517

Roche, N.D., Almaini, O., Dunlop, J.S., Ivison, R.J., Willot, C.J.: 2002, MNRAS 337, 1282

Sadler, E.M., McIntyre, V.J., Jackson, C.A., Cannon, R.D.: 1999, Publications of the Astronomical Society of Australia 16, 247

Simpson, C., Almaini, O., Cirasuolo, M. et al : 2006, MNARS 373, $21 \mathrm{~S}$

Smail, I., Owen, F.N., Morrison, G.E., Keel, W.C., Ivison, R.J, Ledlow, M.J.: 2002, MNRAS 581, 844

Spinrad, H., Dey, A., Stern, D., Dunlop, J., Peacock, J., Jimenez, R., Windhorst, R.: 1997, ApJ 484, 581.

Stockton, A., McGrath, E., Canalizo, G.: 2006, ApJ 650, 706

Takagi, T., Mortier, A.M.J, Shimasaku, K., et al.: 2007, MNRAS 381, 1154

Takata, T., Kashikawa, N., Nakanishi, K. et al.: 2003, PASJ 55, 789

Urry, C.M, Padovani, P.: 1995, PASP 107, 803

Willott, C J., Rawlings, S., Blundell, K.L.: 2001, MNRAS 324, 1

Windhorst, R.A., Miley, G. K., Owen, F.N., Kron, R.G., Koo, R.C. : 1985 ApJ 289, 494

Wold, M., Armus, L., Neugebauer, G., Jarrett, T.H., Lehnert, M.D.: 2003, AJ 126, 1776

Yan, L., Thompson, D.: 2003, ApJ 586, 765

York, D.G., Adelman, J., Anderson, J.E. et al.: 2000, AJ 120, 1579

Zheng, W., Overzier, R., Bouwens, R.J. et al: 2006, AJ 640, 574 
Table 1 Photometric redshift results for ERO sample. Cols (1) Object name, colas(2) flux density of each radio source and (3)and (4) colors; colas(5)-(6): Best-fit photometric redshift, $X_{\nu}^{2}$ for a Gissel 98 elliptical galaxy; colas(7)-(8): Best-fit photometric redshift, $X_{\nu}^{2}$ for a Gissel 98 starburst model; colas(9)-(11): Best-fit photometric redshift, $X_{\nu}^{2}$ when all SED template included (E, burst, SO, Sa, Sb, Sc, Sd, Im) for a Gissel 98 elliptical galaxy.

\begin{tabular}{|c|c|c|c|c|c|c|c|c|c|c|}
\hline & & & & All BC & mplates & Stal & urst & Ell & tical & \\
\hline $\begin{array}{c}\text { Object name } \\
\text { (1) }\end{array}$ & $\begin{array}{c}S_{1.4}(m J y) \\
(2) \\
\end{array}$ & $\begin{array}{c}R-K \\
(3) \\
\end{array}$ & $\begin{array}{c}J-K \\
(4) \\
\end{array}$ & $\begin{array}{c}z \\
(5) \\
\end{array}$ & $\begin{array}{l}\chi_{\nu}^{2} \\
(6) \\
\end{array}$ & $\begin{array}{c}z \\
(7) \\
\end{array}$ & $\begin{array}{l}\chi_{\nu}^{2} \\
(8)\end{array}$ & $\begin{array}{c}z \\
(9) \\
\end{array}$ & $\begin{array}{c}\chi_{\nu}^{2} \\
(10) \\
\end{array}$ & $\begin{array}{l}\text { SED } \\
(11) \\
\end{array}$ \\
\hline NDWFS_J142439.6+3349 & 2.71 & 5.43 & 1.82 & 1.075 & 0.26 & 1.220 & 0.46 & 1.050 & 0.23 & $\mathrm{E}$ \\
\hline NDWFS_J142449.3+3343 & 2.02 & 5.89 & 3.73 & 2.435 & 0.61 & 2.445 & 4.63 & 1.395 & 3.86 & $\mathrm{E}$ \\
\hline NDWFS_J142525.2+3345 & 4.66 & 5.02 & 1.72 & 1.345 & 0.20 & 1.345 & 0.21 & 1.135 & 2.38 & Burst \\
\hline NDWFS_J142611.0+3339 & 1.70 & 5.30 & 2.10 & 0.880 & 0.36 & 1.215 & 1.56 & 1.030 & 1.64 & $\mathrm{SO}$ \\
\hline NDWFS_J142616.8+3309 & 1.09 & 5.46 & 1.65 & 1.800 & 0.85 & 1.800 & 0.85 & 1.255 & 5.97 & Burst \\
\hline NDWFS_J142643.3+3351 & 2.86 & 5.79 & 1.65 & 1.555 & 0.89 & 1.555 & 0.89 & 1.095 & 3.11 & Burst \\
\hline NDWFS_J142755.9+3321 & 16.74 & 5.30 & 1.40 & 1.845 & 1.63 & 1.780 & 8.58 & 1.845 & 1.63 & $\mathrm{E}$ \\
\hline NDWFS_J142843.0+3326 & 5.97 & 5.33 & 1.87 & 1.770 & 6.69 & 1.490 & 34.36 & 1.770 & 6.69 & $\mathrm{E}$ \\
\hline NDWFS_J142843.4+3355 & 2.41 & 5.82 & 4.82 & 2.945 & 3.24 & 2.945 & 3.24 & 2.495 & 18.38 & Burst \\
\hline NDWFS_J142910.3+3358 & 2.24 & 5.72 & 1.31 & 1.740 & 2.14 & 1.740 & 2.14 & 1.305 & 3.13 & Burst \\
\hline NDWFS_J142915.2+3303 & 1.79 & 7.37 & 1.73 & 1.675 & 13.54 & 1.675 & 13.54 & 1.095 & 67.44 & Burst \\
\hline NDWFS_J142916.1+3355 & 2.07 & 5.04 & 1.62 & 1.125 & 1.52 & 1.125 & 1.52 & 0.935 & 3.16 & Burst \\
\hline NDWFS_J143004.7+3302 & 3.18 & 5.61 & 1.60 & 1.580 & 0.46 & 1.580 & 0.46 & 1.095 & 3.58 & Burst \\
\hline NDWFS_J143116.7+3354 & 1.69 & 5.62 & 1.82 & 0.960 & 4.08 & 0.960 & 4.08 & 0.840 & 4.87 & Burst \\
\hline NDWFS_J143209.1+3356 & 1.93 & 5.11 & 2.17 & 0.605 & 2.16 & 0.885 & 4.90 & 0.800 & 4.90 & $\mathrm{E}$ \\
\hline NDWFS_J143226.7+3330 & 4.53 & 5.49 & 1.78 & 1.945 & 0.52 & 1.945 & 0.52 & 1.095 & 4.49 & Burst \\
\hline NDWFS_J143249.7+3316 & 1.32 & 5.23 & 2.33 & 1.005 & 6.91 & 1.005 & 6.91 & 0.840 & 12.61 & Burst \\
\hline NDWFS_J143258.3+3315 & 0.81 & 5.85 & 1.72 & 1.480 & 0.46 & 1.480 & 0.46 & 1.010 & 32.56 & Burst \\
\hline NDWFS_J143304.2+3334 & 2.95 & 5.59 & 1.75 & 1.595 & 99.42 & 1.595 & 99.42 & 1.395 & 174.09 & Burst \\
\hline NDWFS_J143347.0+3353 & 1.19 & 7.34 & 2.22 & 1.700 & 0.00 & 1.700 & 0.00 & 1.395 & 9.54 & Burst \\
\hline NDWFS_J143428.0+3311 & 23.23 & 5.30 & 2.13 & 1.820 & 9.84 & 1.490 & 30.71 & 1.820 & 9.84 & $\mathrm{E}$ \\
\hline NDWFS_J143435.1+3343 & 5.62 & 5.21 & 1.61 & 1.295 & 0.36 & 1.295 & 0.36 & 1.000 & 2.36 & Burst \\
\hline NDWFS_J143527.9+3311 & 39.60 & 6.65 & 2.22 & 1.650 & 1.10 & 1.530 & 16.12 & 1.395 & 7.84 & $\mathrm{E}$ \\
\hline NDWFS_J143646.0+3345 & 3.22 & 6.64 & 3.33 & 2.545 & 0.86 & 2.545 & 0.86 & 1.395 & 8.13 & Burst \\
\hline NDWFS_J143731.0+3340 & 2.64 & 6.46 & 1.64 & 1.650 & 1.20 & 1.650 & 1.20 & 1.385 & 12.89 & Burst \\
\hline NDWFS_J142517.2+3415 & 14.74 & 5.00 & - & 1.580 & 8.18 & 1.580 & 8.18 & 1.100 & 14.74 & $\mathrm{E}$ \\
\hline NDWFS_J142648.2+3458 & 4.46 & 5.70 & - & 1.305 & 0.25 & 1.020 & 1.20 & 1.305 & 0.25 & Burst \\
\hline NDWFS_J142802.4+3437 & 1.20 & 6.49 & - & 1.105 & 2.85 & 1.030 & 3.87 & 1.105 & 2.86 & Burst \\
\hline NDWFS_J142850.6+3453 & 2.19 & 5.75 & - & 1.015 & 0.11 & 0.840 & 3.29 & 1.015 & 0.11 & Burst \\
\hline NDWFS_J142905.6+3449 & 31.42 & 5.04 & - & 1.355 & 0.23 & 1.045 & 2.14 & 1.355 & 0.22 & Burst \\
\hline NDWFS_J142917.6+3437 & 2.56 & 5.25 & - & 0.840 & 3.40 & 0.805 & 5.33 & 0.840 & 3.39 & Burst \\
\hline NDWFS_J142943.8+3434 & 1.89 & 6.00 & - & 1.300 & 0.03 & 1.035 & 1.53 & 1.300 & 0.03 & Burst \\
\hline NDWFS_J143248.7+3413 & 0.95 & 5.25 & - & 0.555 & 0.05 & 0.735 & 0.72 & 0.555 & 0.05 & Burst \\
\hline NDWFS_J143259.2+3406 & 1.14 & 6.56 & - & 1.125 & 0.41 & 1.190 & 1.19 & 1.385 & 1.56 & $\mathrm{E}$ \\
\hline NDWFS_J143308.0+3418 & 7.07 & 5.10 & - & 0.805 & 2.45 & 0.795 & 6.11 & 0.805 & 2.44 & Burst \\
\hline NDWFS_J143430.5+3427 & 1.50 & 5.81 & - & 1.430 & 1.24 & 1.430 & 1.24 & 1.125 & 5.92 & $\mathrm{E}$ \\
\hline NDWFS_J143506.4+3438 & 3.22 & 5.16 & - & 0.935 & 8.67 & 0.840 & 13.65 & 0.935 & 8.67 & Burst \\
\hline NDWFS_J143539.8+3443 & 23.22 & 5.74 & - & 0.780 & 0.21 & 0.830 & 6.64 & 0.895 & 0.22 & $\mathrm{E}$ \\
\hline NDWFS_J142440.4+3511 & 1.06 & 6.11 & - & 1.850 & 0.10 & 1.425 & 13.21 & 1.625 & 23.32 & $\mathrm{E}$ \\
\hline NDWFS_J142618.6+3545 & 2.49 & 5.56 & - & 1.860 & 0.02 & 1.070 & 1.14 & 1.860 & 0.02 & Burst \\
\hline NDWFS_J142628.5+3527 & 1.79 & 5.39 & - & 2.495 & 5.16 & 0.275 & 9.12 & 2.210 & 6.36 & $\mathrm{E}$ \\
\hline NDWFS_J142639.3+3510 & 2.56 & 5.18 & - & 0.850 & 0.10 & 0.870 & 0.00 & 1.070 & 0.20 & $\mathrm{E}$ \\
\hline NDWFS_J142817.2+3509 & 5.46 & 6.31 & - & 0.805 & 4.41 & 0.940 & 9.10 & 0.805 & 4.41 & Burst \\
\hline NDWFS_J143042.5+3512 & 5.18 & 5.23 & - & 2.495 & 17.63 & 0.260 & 20.76 & 2.415 & 18.45 & $\mathrm{E}$ \\
\hline NDWFS_J143051.3+3543 & 3.41 & 5.51 & - & 0.805 & 0.39 & 0.840 & 1.04 & 0.805 & 0.40 & Burst \\
\hline NDWFS_J143108.3+3525 & 2.07 & 5.36 & - & 1.335 & 0.07 & 1.020 & 0.32 & 1.335 & 0.07 & Burst \\
\hline NDWFS_J143112.5+3535 & 9.64 & 6.08 & - & 1.405 & 0.02 & 0.840 & 9.08 & 1.405 & 0.02 & Burst \\
\hline NDWFS_J143134.5+3515 & 64.12 & 5.94 & - & 0.800 & 0.00 & 1.010 & 1.28 & 0.800 & 0.00 & Burst \\
\hline NDWFS_J143238.0+3530 & 17.86 & 5.20 & - & 0.905 & 0.27 & 0.790 & 1.85 & 0.905 & 0.27 & Burst \\
\hline NDWFS_J143246.7+3533 & 2.18 & 5.28 & - & 1.825 & 0.05 & 0.605 & 6.61 & 1.825 & 0.05 & Burst \\
\hline NDWFS_J143313.7+3539 & 0.90 & 6.04 & - & 0.750 & 0.01 & 1.030 & 2.01 & 0.750 & 0.01 & Burst \\
\hline NDWFS_J143414.5+3541 & 0.55 & 5.95 & - & 1.240 & 0.00 & 1.240 & 0.00 & 1.495 & 0.83 & $\mathrm{E}$ \\
\hline
\end{tabular}

